\title{
Vulnerability assessment modelling for railway networks
}

\author{
R. Meesit* and J. Andrews** \\ Resilience Engineering Research Group, University of Nottingham, University Park, Nottingham, NG7 2RD \\ *ratthaphong.meesit@nottingham.ac.uk**john.andrews@nottingham.ac.uk
}

\begin{abstract}
Railway networks are prone to many different potential disruptive events such as technical failures (e.g. the failure of aging components), natural disasters (e.g. flooding) and intentional man-made disasters (e.g. trespass and suicide). Assessing the vulnerability of railway networks can help infrastructure managers to create the right preventive strategies to improve the robustness and the resilience of railway networks before the occurrence of disruptions. This study proposes a stochastic-vulnerability analysis model that enables the critical components of railway networks to be identified. The model is developed using a discrete event simulation technique. Its framework includes modules for assigning the disruption to the network components, predicting the network vulnerability, in terms of passenger delays and journey cancellations, and calculating the risk-based criticality of network components. Finally, an example application of the model is presented using a part of the East Midland railway network in UK.
\end{abstract}

\section{Introduction}

Over the past decade, railway networks have been significantly impacted by many different disruptive events such as technical failures and natural disasters. These events are difficult to predict, and some specific instances, such as flooding and earthquakes, are impossible to prevent. Thus, to avoid service disruptions the railway needs to be able to counter such occurrences. The robustness and the resilience of a railway network must be improved in order to maintain services and serve public demand during disruptions (Cabinet Office, 2010). To this end, networks would need to be enhanced to accommodate disruptions. Changes to the network are however limited through physical barriers and budget constraints. Infrastructure managers are required to make a decision on the effectiveness and priority of any improvements or added protection. This generates a need to develop a model that can support infrastructure managers in creating suitable prevention strategies to reduce the vulnerability of railway networks.

In recent years, various vulnerability analysis models have been proposed for railway networks. The majority of the initial models were based on the concept of complex network theory and used to assess the vulnerability of railway systems. For instance, Zhang et al. (2011) used complex network theory to investigate the reliability and the robustness of the Shanghai subway network. The global functionality loss (i.e. the combination of the degree of a node and the shortest path length between a node and the others) and the connectivity of railway lines (i.e. the connection of nodes on the impacted railway line) were calculated as the indicators of network vulnerability. The application of other topological indicators, such as global efficiency (i.e. the ability of a network to transfer the flow) and maximal connected subgraph (i.e. the maximum number of connected nodes in the network after disruptions), can be seen in the study of Deng et al. (2013).

Using only complex network analysis models to quantify vulnerability seems to be a useful method from the network structural point of view. However, it does not reflect the functional vulnerability of railway networks. For this reason, more recent attention has focused on estimating network flows of trains and passengers as the indicators of network vulnerability. In terms of the train flow, Ouyang et al. (2014) introduced a train flow model for vulnerability quantification. The model was constructed by considering the number of trains operating daily on each link. When a disruption happened (i.e. node removal), the accessibility of stations and the number of trains that can still operate, were calculated for the analysis. Hong et al. (2015) presented a model to evaluate the vulnerability of railway networks to flooding events. The effect of flooding events on network components was simulated using a Monte-Carlo simulation. Then, the method proposed by Ouyang et al. (2014) was adapted to estimate the performance of the railway network during flooding events.

For passenger flow, Zhang et al. (2015) adapted a gravity model to predict the movement of passengers between stations in order to evaluate the criticality of railway links. Sun et al. (2015) constructed a passenger flow model based on the passenger Origin and Destination ratio to investigating station vulnerability. Some passenger movement characteristics such as interchange times and route selections (i.e. in terms of travel time) 
were included. The vulnerability indicators were passenger flow influence (i.e. flow volume affected by disruptions) and platform passenger flow (i.e. passenger volume at station during disruptions). In addition, a comprehensive rail passenger simulation model was developed by Pant et al. (2016) for assessing the vulnerability of a large-scale railway network. The model can estimate passenger trips lost during disruptions. The interdependency between a railway system and other infrastructures such as electricity substations and telecommunication equipment was analysed, and disruptions resulting from the failures of these different infrastructures and flood likelihoods in UK were modelled.

As can be seen from the literature, these vulnerability analysis models were mainly developed for identifying the critical components of railway networks and used complex network theory to construct macroscopic models. This type of model has advantages that they are reasonably simple to apply on a largescale railway network and can also provide a quick solution for network robustness improvement. However, this method only pays attention to the analysis of a network topology. Even though recent studies have attempted to take into account some railway properties such as train and passenger flow, the results obtained are approximations since they do not consider other significant characteristics of railway networks such as the traffic control systems, the conditions of train movements and passenger behaviour when a disruption occurs.

The aim of this study is to introduce the use of a stochastic microscopic simulation model to assess the vulnerability of railway networks. This detailed modelling approach is able to predict the system performance of railway networks during disruptions and identify the critical components of railway networks in terms of risk-based criticality. These capabilities will enable infrastructure managers to establish the priority for which enhancement features are incorporated into the railway.

\section{Railway network performance estimation}

The railway network simulation model (Meesit and Andrews, 2017) is applied in this study to estimate the key performance indicators (KPIs) of railway networks (e.g. train delays, service cancellations, passenger delays and passenger journey cancellations) during disruptions. The model was developed using a stochastic-discrete event simulation technique. Its framework consists of two main modules: railway network modelling and passenger modelling. The description of each module is presented as follows.

\subsection{Railway network modelling}

The first module comprises three submodules: infrastructure module, control system module and operational module. The infrastructure module represents the railway network infrastructure assets such as track sections, stations and junctions. These infrastructure items are modelled as static resources with their significant attributes such as block lengths and speed restrictions. A state is then assigned to describe the possible status of each resource. Each of their states are considered as a set of mutually exclusive and exhaustive variables such as "occupied or available" and "working or failed". The control system module creates train movement conditions accounting for the state of the block sections. For example, if the next two blocks are available (Green), a train can run to the next block at the permitted speed or at full operational speed. If only one block ahead is available (Yellow), a train needs to reduce its speed and prepare to stop at the next signal. However, if the block ahead is unavailable (Red), a train must stop and wait until it is free. Finally, the operational module produces the input data required for the simulation. Three main aspects (trains, service routes, and timetables) are considered. Trains are modelled as dynamic objects running on the network. The service routes of trains are modelled using the order of track section IDs, and for timetables, only departure times at terminal stations are required for the model. Then, the detailed timetables of train arrivals and departures at each station (in both normal and disruptive situation) are generated during the simulation and used as passenger information.

\subsection{Passenger movement modelling}

Passenger movement modelling is separated into three main parts: passenger arrivals at a station, passengers alighting/boarding a train and passenger behaviour during a disruption. Passenger arrivals at a station are simulated by a Poisson process. Then, the number of passenger arrivals is distributed to each destination station based on an origin-destination (O-D) matrix and saved into a passenger object which is created to contain the significant information about passengers such as the number of passengers, arrival time at origin, expected 
arrival time at destination and the best route choice (i.e. route vector). This passenger object is properly stored in the passenger vector at an origin station waiting for a train to go to a destination. Passengers alighting and boarding happens when a train is at a station. Passengers alight from a train if the first destination in a route vector matches with the station. If a station is an interchange station, passengers alight from a train and wait for their connection. However, if a station is the final destination, passengers alight from a train and leave the network. Then, by comparison with the scheduled timetable, passenger delays are collected. Boarding occurs after passengers leaving the train have alighted. Passengers at a station compare their destination with the calling stations of the train. If their destination matches those of the trains calling stations, passengers then get on the train. Otherwise, they must wait for another train. It is noted that all trains and stations are modelled using passenger vectors. Thus, these processes are simulated by moving the passenger objects between the passenger vectors of stations and trains. The movements of passenger objects are limited by the capacity of stations and trains. Finally, passenger behaviour during a disruption is simulated in the model as presented in Figure 1. Before the occurrence of a disruption, all passengers use a normal timetable as travel information. Once a disruption happens, passengers are assumed to travel based on the disrupted timetable. A disrupted timetable refers to information provided to passengers during a disruption (e.g. real-time information announced at stations or displayed on smartphones). During this period, existing passengers on the network reconsider their routes according to the new information. If no route is found or the expected travel time is longer than the acceptable travel time (set in the model), passengers cancel their journeys. After recovering from a disruption, passengers then return to using the normal timetable.

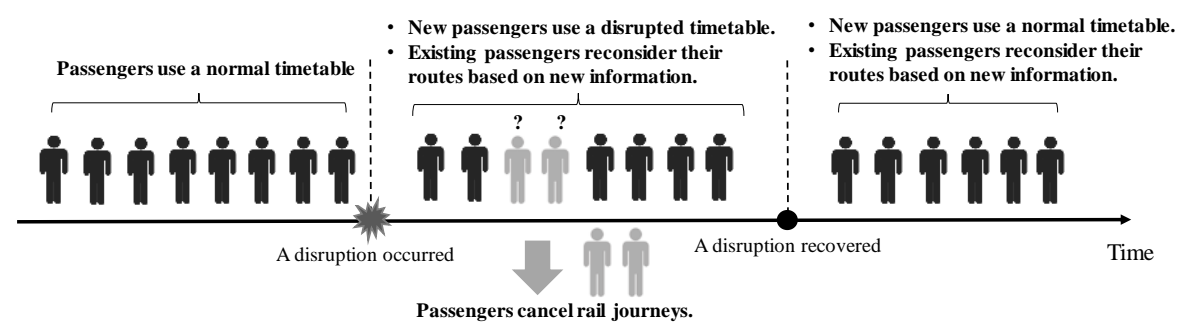

Figure 1. Passenger behaviour during a disruption simulated in the model.

\section{Railway network vulnerability analysis model}

The main purpose of the vulnerability analysis model is to identify the critical components of the railway network (e.g. stations or railway links). A critical component is defined as a component that, if it fails, causes large negative impacts on the network (Jönsson et al., 2008). Thus, to achieve the aim, three main steps are proposed: assigning a disruption, assessing vulnerability, and evaluating the risk-based criticality. These steps are described as follows.

\subsection{Assigning a disruption to tested components}

A disruptive event is simulated by changing the state of the failed network component to 'unavailable'. This affects the movement conditions set, as described in Section 2.1, resulting in a line blockage. Thus, to conduct a vulnerability analysis, a disruption is assigned to each testing component one at a time. Either a short duration incident (less than $1 \mathrm{hr}$.) or a large impact disruption (more than $1 \mathrm{hr}$.) can be used in a test. However, only a large impact disruption is considered in this study to demonstrate the model. Then, the railway network simulation is applied to estimate the vulnerability of the railway network due to the section failure disruption.

\subsection{Assessing the vulnerability of a railway network}

As described in Section 2, four KPIs of the railway network, (train delays, train service cancellations, passenger delays and passenger journey cancellations) are predicted during a simulation. However, in order to evaluate the vulnerability of railway networks from the perspective of rail users, there is a focus on the passenger delays and journey cancellations. Passenger delay is defined as the difference between the actual arrival time $\left(A T_{p}\right)$ and the expected arrival time $\left(E T_{p}\right)$ of a passenger $(p)$ at a destination station. The summation of all passenger delays over the network $(P D)$ can be calculated using Equation 1.

$$
P D=\Sigma_{p}^{P}\left(A T_{p}-E T_{p}\right)
$$


Passenger journey cancellations $(P C)$ are defined as the number of passengers who cancel their journeys when the delay time exceeds the defined threshold. This number is collected and converted to equivalent passenger delays using a weighting factor $(w)$. This is then incorporated into Equation 1 to obtain the total passenger delays which is used as the vulnerability indicator, $V$ (see Equation 2).

$$
V=P D+P C(w)
$$

where: $w$ is the weighting factor to convert passenger journey cancellations to equivalent delay minutes. In this study it is assumed that 1 passenger journey cancellation is equal to 60 -minute passenger delays $(w=60)$.

\subsection{Evaluating risk-based criticality}

The risk-based criticality $(R C)$ of a network component is defined as the product of the vulnerability and the frequency of the network section disruption (historical data). The frequency data can be specific to events of interest such as flooding or landslides, if the focus is on a particular disruption type, or it can be the overall frequency from all disruptive events on the railway link (useful for setting annual improvement budgets). In this study, RC is applied to prioritise the criticality of railway network section. Sections with the higher predicted RC are used to prioritise infrastructure manager actions.

\section{Model Application}

\subsection{Case study}

A part of the East Midland railway network is used to demonstrate the application of the model. The network consists of 12 stations and 15 links (double track). The total length of the network is approximately $58 \mathrm{~km}$ as displayed in Figure 2(a). In addition, it is assumed there are 3 services operate daily from 6:00 to 22:00. The first service (S1) operates between Derby and Nottingham with the frequency of 1 train per hour. The other two services operate between Derby and Leicester (S2) and Nottingham and Leicester (S3) with a frequency of 2 trains per hour. All trains on the network are the British rail class 158 (3 coaches), and they stop at every intermediate station along their routes. For passengers, the peak travel hours are 7:00 to 10:00 and 16:00 to 19:00. The acceptable delay of passengers was set to 45 minutes. Passengers arrive at each station at different mean arrival rates. Then, they are distributed to their destinations based on the O-D matrix of passengers input to the model based on historical journey patterns indicated through ticket sales. An example of the number of passenger arrivals at a station (Attenborough) from a simulation is depicted in Figure 2(b).

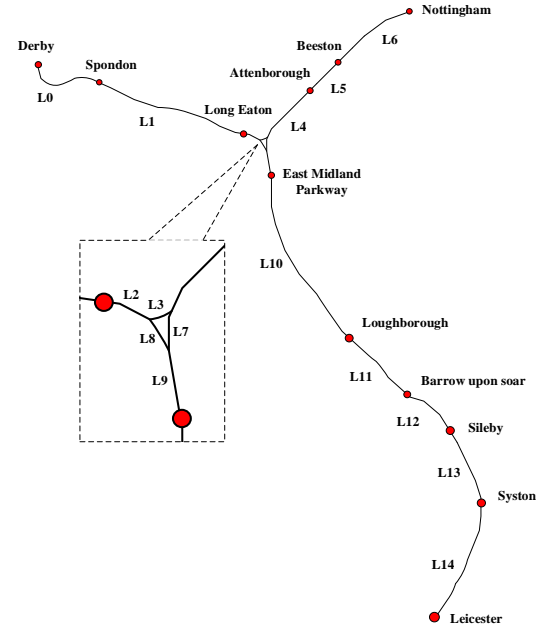

(a) A part of the East Midland railway network

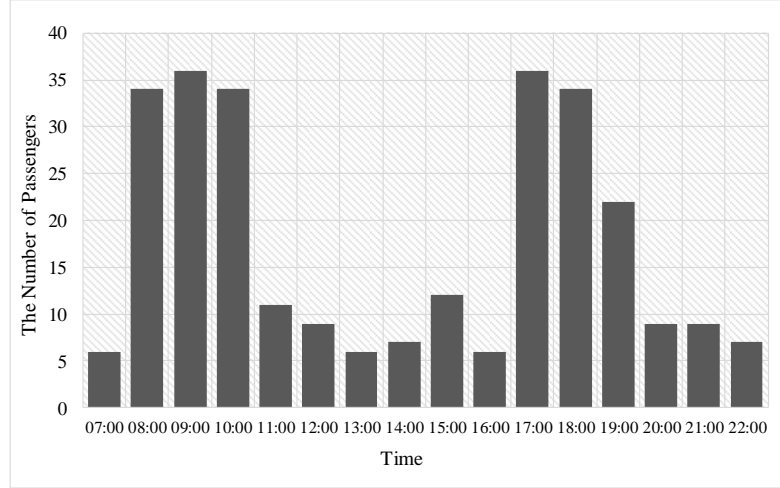

(b) The Attenborough station

Figure 2. A tested network (a) and the number of passenger arrivals at the station from a simulation (b).

\subsection{Results}

The evaluation of the link criticality is presented as an example in this study. The model software was developed in $\mathrm{C}++$. The effects of large impact disruptions with mean durations of 5,10 and $24 \mathrm{hrs}(\mathrm{SD}=0.5$, 1 and 2 hrs respectively) were considered with occurrence times of 13:00, 13:15, 13:30 and 13:45. Then, the average vulnerability of each link was calculated from the results of 2,000 simulations (at which point convergence had occurred). 


\subsubsection{The vulnerability of the railway links}

Figures 3(a), 3(b) and 3(c) present the results obtained from running the model. It is found that the occurrence times of the large impact disruptions has little effect on the vulnerability prediction. Even though the vulnerability of each link was slightly different when the occurrence time was changed, there was no significant difference in the rank of the railway link criticality. Therefore, any large impact disruptions can be applied and considered representative when performing the railway link criticality analysis.

Based on the findings, the study selected a test using a $24 \mathrm{hr}$-disruption for the analysis. The averaged vulnerability of each railway link resulting from the different occurrence times was calculated. After that, the highest vulnerability of the railway link was normalised to 1 . Then, the vulnerability of other links was specified as its proportion of the highest value. This method allows the vulnerability of the railway links to be presented in the range of 0 to 1 as shown in Figure 3(d).

In Figure 3(d), Links 9, 10, 11, 12, 13 and 14 were all high criticality links on this network. Their vulnerabilities were approximately $43 \%$ higher than the next group of critical links, which are links $0,1,2,4$, 5 and 6 . The least critical links of this network are those located at the junction (e.g. links 3, 7 and 8). This is because if one of these links is down, it affects only one of the three service routes. Thus, it is still possible for passengers to travel to all stations on the network, but their journey times will increase significantly due to the limited availability of the train services on the network.

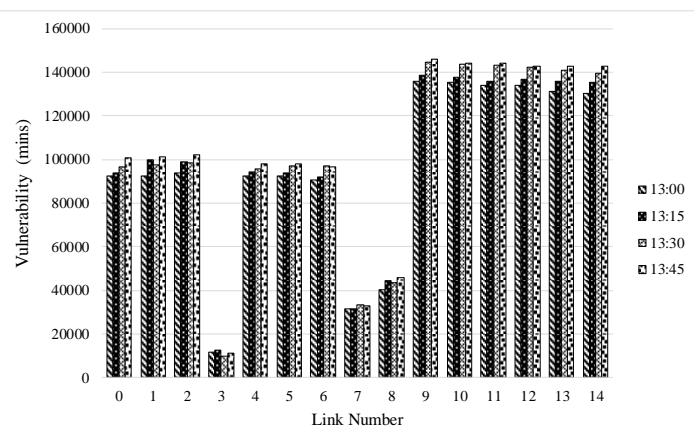

(a) Testing with $5 \mathrm{hr}$-disruption.

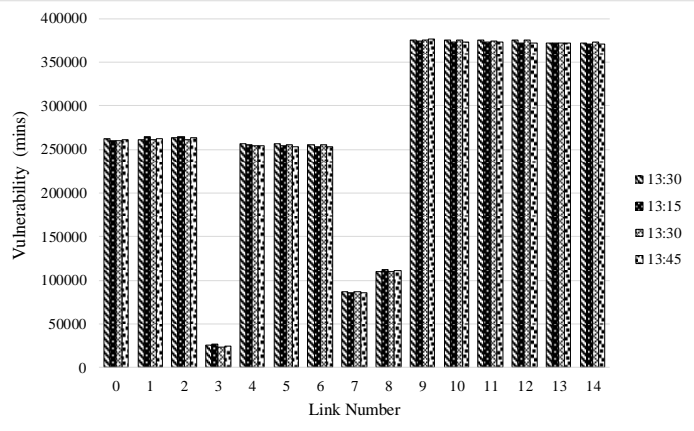

(c) Testing with $24 \mathrm{hr}$-disruption.

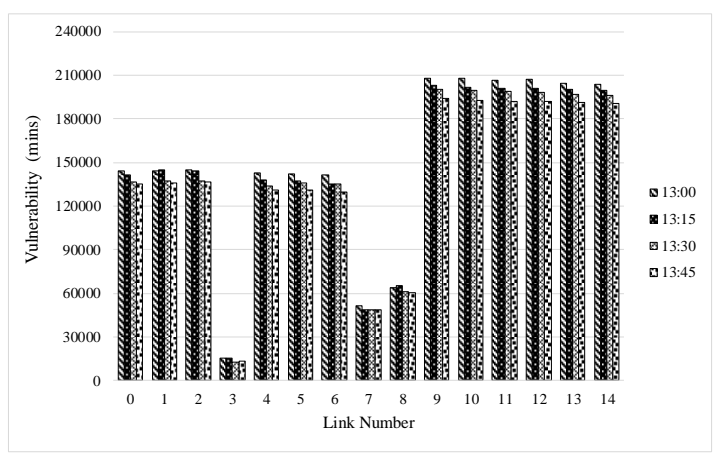

(b) Testing with $10 \mathrm{hr}$-disruption.

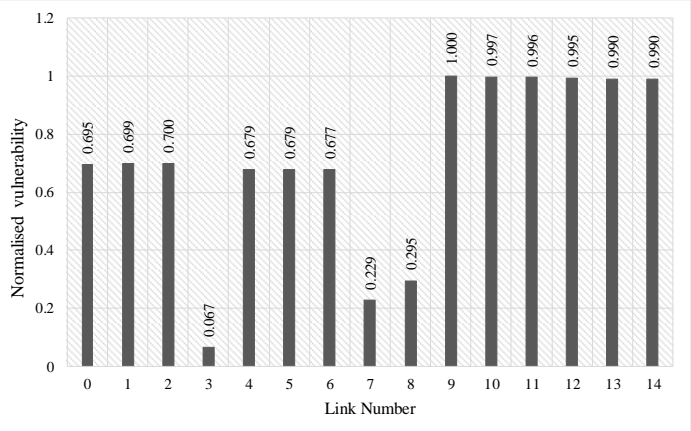

(d) The normalised vulnerability of each railway link

Figure 3. The vulnerability of each railway link.

\subsubsection{The risk-based criticality of the railway links}

The risk-based criticality of each railway link was estimated by using the normalised vulnerability and the overall frequency of the railway link disruptions from the past 10 years. To prove the concept of the model, this frequency data was reasonably assumed by considering the three main causes of disruptions on the railway network: technical failures (e.g. track failure, signaling failure and bridge collapse), natural disasters (e.g. flooding, landslide/landslip, and fallen tree) and man-made disasters (e.g. accident, trespass and suicide). Only the large impact disruptions were taken into account (See Table 1).

Table 1 illustrates the risk-based criticality of each railway link on the tested network. Link 11 is predicted as the most critical link on this network. Its risk exposure is 6.970 which represents an increase of $16.53 \%$ compared to the second critical link (i.e. link 10). The least critical link on this network was link 3 which has a risk exposure of 0.337 . Therefore, based on these results, the limited budgets available for enhancing the robustness of the railway network would be most productively directed towards link 11 . This would be the top priority, and link 3 would be the least priority that requires improvement for protection. 
Table 1 . The risk-based criticality of the railway links on the network.

\begin{tabular}{cccccc}
\hline \multirow{2}{*}{$\begin{array}{c}\text { Link } \\
\text { No. }\end{array}$} & \multicolumn{2}{c}{ The frequency of railway link disruptions } & & $\begin{array}{c}\text { Risk-based } \\
\text { criticality }\end{array}$ \\
\cline { 2 - 5 } & Technical failures & Natural disasters & Man-made disasters & Total & 2.781 \\
1 & 3 & 0 & 1 & 4 & 3.495 \\
2 & 3 & 2 & 0 & 5 & 2.101 \\
3 & 2 & 0 & 1 & 3 & 0.337 \\
4 & 5 & 0 & 0 & 5 & 4.076 \\
5 & 4 & 1 & 1 & 6 & 2.716 \\
6 & 3 & 0 & 1 & 4 & 3.385 \\
7 & 5 & 0 & 0 & 5 & 1.147 \\
8 & 5 & 0 & 0 & 5 & 1.772 \\
9 & 6 & 0 & 0 & 6 & 5.000 \\
$\mathbf{1 0}$ & 4 & 1 & $\mathbf{1}$ & 5 & $\mathbf{5 . 9 8 1}$ \\
$\mathbf{1 1}$ & $\mathbf{4}$ & $\mathbf{1}$ & 1 & $\mathbf{6}$ & $\mathbf{6 . 9 7 0}$ \\
12 & $\mathbf{4}$ & $\mathbf{2}$ & 0 & 5 & 4.975 \\
13 & 4 & 0 & 1 & 3 & 2.971 \\
14 & 3 & 0 & & 4 & 3.961 \\
\hline
\end{tabular}

\section{Conclusion}

A stochastic railway network vulnerability assessment model is presented in this paper. The model applied two vulnerability indicators: passenger delays and passenger journey cancellations to identify the critical section links of a railway network. The application of the model was demonstrated using a section of the East Midland railway network, and the assessment of railway link criticality using different large impact disruptions was performed. In the future, the model will be further developed to investigate the criticality of other network components (e.g. stations and points). Other KPIs (e.g. train delays and train service cancellations) will also be used and compared. Then, the vulnerability analysis conducting with small impact disruptions will be studied, and the more detail about network component disruptions will be considered using a fault tree approach. This will enable the model to provide better results about network component criticality in both a qualitative and quantitative point of view.

\section{Acknowledgements}

Ratthaphong Meesit is in receipt of a PhD scholarship to study at University of Nottingham from the Royal Thai Government. Professor John Andrews is the Royal Academy of Engineering and Network Rail Chair in Infrastructure Asset Management. Both authors would like to gratefully acknowledge these organisations.

\section{References}

Cabinet Office. (2010). Strategic framework and policy statement on improving the resilience of critical infrastructure to disruption from natural hazards. London, UK.

Deng, Y., Li, Q., Lu, Y., Yuan, J. (2013). Topology vulnerability analysis and measure of urban metro network: The case of Nanjing. Journal of Networks, 8(6), 1350-1356.

Hong, L., Ouyang, M., Peeta, S., He, X., Yan, Y. (2015) Vulnerability assessment and mitigation for the Chinese railway system under floods. Reliability Engineering and System Safety, 137, 58-68.

Jönsson, H., Johansson, J., Johansson, H. (2008) Identifying critical components in technical infrastructure networks. Proc. The Institution of Mechanical Engineers, Part O: Risk and Reliability, 222(2), 235-243.

Meesit, R., Andrews, J. (2017) Railway network resilience modelling. Transportation Research Part B: Methodological, Submitted.

Ouyang, M., Zhao, L., Hong, L., Pan, Z. (2014) Comparisons of complex network based models and real train flow model to analyze Chinese railway vulnerability. Reliability Engineering and System Safety, 123, 38-46.

Pant, R., Hall, J. W., Blainey, S. P. (2016) Vulnerability assessment framework for interdependent critical infrastructures: case-study for Great Britain's rail network. European Journal of Transport and Infrastructure Research, 16(1), 174-194.

Sun, D., Zhao, Y. \& Lu, Q.-C. (2015) Vulnerability analysis of urban rail transit networks: A case study of Shanghai, China. Sustainability, 7(6), 6919-6936.

Zhang, J., Xu, X., Hong, L., Wang, S., Fei, Q. (2011) Networked analysis of the Shanghai subway network, in China. Physica A: Statistical Mechanics and its Applications, 390, 4562-4570.

Zhang, Z., Li, X., Li, H. (2015) A quantitative approach for assessing the critical nodal and linear elements of a railway infrastructure. International Journal of Critical Infrastructure Protection, 8, 3-15. 